\title{
Study on the Spatial Differentiation Pattern of Housing Prices in Chengdu-Chongqing City Cluster
}

\author{
Renkang Feng*, Rubing Han \\ School of Civil Engineering and Architecture, Southwest University of Science and Technology, Mianyang, China \\ Email: ^1216467516@qq.com
}

How to cite this paper: Feng, R.K. and Han, R.B. (2021) Study on the Spatial Differentiation Pattern of Housing Prices in ChengduChongqing City Cluster. Open Access Library Journal, 8: e7118.

https://doi.org/10.4236/oalib.1107118

Received: December 28, 2020

Accepted: February 23, 2021

Published: February 26, 2021

Copyright $\odot 2021$ by author(s) and Open Access Library Inc.

This work is licensed under the Creative Commons Attribution International License (CC BY 4.0).

http://creativecommons.org/licenses/by/4.0/

\begin{abstract}
Taking the housing prices of 151 districts and counties in the Chengdu-Chongqing urban agglomeration from 2017 to 2019 as the basic data, the housing price data is geospatially expressed in ArcGIS, and the Theil index is calculated to analyze the differentiation pattern of the housing prices of the Chengdu-Chongqing urban agglomeration, using geo-detector. The effect of different factors on housing prices in different regions shows that the geographical distribution of housing prices in the Chengdu-Chongqing urban agglomeration is basically the same as the city scale and economic development of each city. The housing prices of the Chengdu-Chongqing urban agglomeration show a dual-core dominance and a collapse in the central region. The characteristics of industrial structure, housing supply, population scale, land cost, economic strength, real estate investment scale, housing policy, and higher education resources have a significant impact on the housing prices of Chengdu-Chongqing urban agglomeration. Different indicators have different effects on housing prices in different cities.
\end{abstract}

\section{Subject Areas}

Development Economics, Urban Planning

\section{Keywords}

Chengdu-Chongqing Urban Agglomeration, Housing Price, Spatial

Differentiation

\section{Introduction}

Since the abolition of the welfare distribution housing system and the implementation of housing distribution monetization reform in 1998, the real estate 
industry has developed rapidly in my country. The contribution rate of the real estate industry and its affiliated industries to my country's GDP growth once reached $30 \%$ [1], especially after the real estate industry became the pillar industry of the national economy in 2003, its development has become unstoppable in the past 20 years. My country has a vast territory. With the development of real estate coverage across the country, the development of real estate in different cities and regions is very different. The most direct manifestation is the heterogeneity of urban housing prices in different spatial and geographic locations. In return, this phenomenon has increased the incoordination of regional development. Therefore, studying the spatial differentiation of housing prices in different regions will not only help promote the coordinated development of regions, but also benefit the healthy and reasonable development of real estate.

Regarding the issue of spatial differentiation of housing prices, scholars at home and abroad have studied it from different angles. Sabyasachi Basu and Thomas GThibodeau studied the spatial autocorrelation effect in the transaction prices of single-family residential properties in Dallas, Texas, and the results showed that there is a spatial autocorrelation phenomenon in housing transaction prices in different regions [2]. Dean M. Hanink et al. conducted a study on the county-level residential real estate market in China, and the results showed that structural convenience and location environment have a great influence on the spatial differentiation of housing prices [3]. Mark J. Holmes et al. studied the degree of difference in housing prices between England and Wales from the perspective of the heterogeneity and convergence of housing prices. The results showed that distance, income, population density, traffic conditions, and education are important factors in the formation of housing price differentiation [4]. In domestic related research, many scholars have studied the influencing factors of urban housing price differentiation of more developed cities such as Beijing [5], Shanghai [6], and Guangzhou [7], from the aspects of rail transit, location factors, distance from the $\mathrm{CBD}$, population structure, infrastructure construction, etc. Another type of research is to analyze the differentiation mechanism of housing prices at the entire region, urban agglomerations and even at the national level, such as the Yangtze River Delta urban agglomeration [8], Chinese prefecture-level city administrative units [9], China County-level administrative units [10] discussed the spatial difference characteristics and influencing factor systems of housing prices. This type of research focuses on the study of macro-factors between cities or within urban agglomerations [11]. The first type of research on housing prices in a certain city alone is relatively common, and the research objects are often targeted at the southeast coast or other more developed and popular cities. The second type of research is less related to regions, and housing prices research based on undeveloped cities in the west areas in China is also very scarce. The Chengdu-Chongqing urban agglomeration is one of the main components of the Yangtze River Economic Belt, a microcosm of the development of urban agglomerations in the western region, and an important driving force leading the high-quality development of the western region 
[12]. However, the distribution of natural geographical factors, labor, technology, capital and other resources in the Chengdu-Chongqing urban agglomeration has huge differences. The development of the Chengdu-Chongqing area is extremely uncoordinated. The evolution of housing prices in the Chengdu-Chongqing urban agglomeration also shows strong spatial heterogeneity. The study of the spatial differentiation and influencing factors of housing prices between and within regions from the level of urban agglomerations is of great significance for the healthy and reasonable development of real estate in Chengdu and Chongqing and the formulation of relevant policies.

\section{Research Methods and Data Sources}

\subsection{Overview of the Study Area}

The Chengdu-Chongqing urban agglomeration is the most competitive urban agglomeration in western China, and it is the backbone of the coordinated development of the western region. From the perspective of urban economy, the Chengdu-Chongqing city clusters mainly include: Chengdu, Mianyang, Deyang, Meishan, Leshan, Yibin, Zigong, Ziyang, Neijiang, Suining, Nanchong, and Dazhou in Sichuan There are a total of 37 districts (cities, counties) including 9 districts of the city, Guang'an City, Luzhou City, the main city of Chongqing and Hechuan, Yongchuan, Nanchuan and Dianjiang. In 2019, the economic zone has a land area of approximately 185,000 square kilometers, a resident population of 95 million, and a regional GDP of 6.4 trillion yuan, accounting for $6.8 \%$ and $6.4 \%$ of the country respectively, forming "two circles", "two groups", The distribution pattern of the "Three Belts".

\subsection{Data Sources}

The house price in the article is the average transaction price of the building area of a commercial housing unit. The basic research unit is 151 district and county-level administrative units in 15 cities in the research area. The housing price data comes from real estate information trading platforms such as Anjuke and China House Price Market. This article collected monthly data on the average price of second-hand housing transactions in 151 districts and counties in the study area from 2017 to 2019. These data are all listed prices in the second-hand real estate market for the month, which can more truly reflect the real level of housing prices in the local area. In view of the fact that the factors that cause housing price differences have commonalities and individualities in different regions, in order to ensure the accuracy and comprehensiveness of the research, combined with the development characteristics of the Chengdu-Chongqing urban agglomeration, combined with related literature, this article selects Economic strength, population size, real estate investment scale, higher education resources, housing supply, house purchase policy, land cost, industrial structure 8 influencing indicators to study the effect of different factors on housing prices in different regions, the relevant data comes from the cities in the study area National Economic and Social Development Statistical Bulletins and Statistical Yearbooks. 


\subsection{Research Method}

\subsubsection{Theil Index Measurement}

The Theil index is an important indicator used to analyze the difference in income levels and inequality between individuals or between regions. Its principle is to use the concept of entropy in information theory to calculate inequality or difference. The Theil index as a measure of inequality has a decomposable nature, that is, when the research object has multiple groups, the research area is divided into different groups, and the Theil index can measure the gap between groups and between groups at different spatial scales gap. This paper uses the decomposed Theil index calculation formula to measure the degree of housing price differentiation in the Chengyu urban agglomeration. The decomposed Theil index calculation formula is as follows:

$$
T=T_{b}+T_{w}=\sum_{k=1}^{k} \frac{x_{k}}{y_{k}} \ln \left(\frac{x_{k} / y_{k}}{n_{k} / n}\right)+\sum_{k=1}^{k} \frac{x_{k}}{y_{k}} \ln \left(\sum_{i \in g_{k}}^{k} \frac{x_{i}}{y_{k}} \ln \frac{x_{i} / x_{k}}{1 / n_{k}}\right)
$$

In the formula (1), $T_{b}$ and $T_{W}$ are the gap between groups and the gap within groups, respectively, and $\mathrm{k}$ represents the number of groups after the study area is divided. In this paper, $k=15, n$ is the number of all districts and counties, and $n=151$ in this paper. The number of districts and counties in the $k$-th group $g_{k}$ is $n_{k}, X_{i}$ is the housing price of the $i$-th district and county, $x_{k}$ is the sum of all regional housing prices in the $k$-th group, and $y_{k}$ is the sum of all districts and counties.

\subsubsection{Principle of Geodetector}

Geodetector is a set of statistical methods to detect spatial differentiation and reveal the driving force behind it. Geodetector $q$ statistics can be used to measure spatial differentiation, detect explanatory factors, and analyze the interaction relationship between variables. And social science multi-domain applications, since there are many influencing factors on housing prices, and the strength of each factor on housing prices in different regions is different, geographic detectors are used to quantitatively calculate the strength of each influencing factor on housing price differences, revealing the direct driving force behind the geographic spatial differentiation of housing prices. The principle is: the various factors that affect housing prices are heterogeneous in space and geography. If a certain factor and housing price changes show consistency or similarity in space, this indicates that this factor has a positive determinant effect on urban housing prices. The calculation principle of the detector is [13]:

$$
q_{D, U}=1-\frac{1}{n \sigma^{2} \mu} \sum_{i=1}^{m}\left(n_{D, i} \sigma_{U D, i}^{2}\right)
$$

In the formula (2), $q_{D, U}$ is indicator of the intensity of the impact of a certain detection factor $\mathrm{D}$ on housing prices, $\mathrm{n}$ is the number of administrative regions in the first-level region of the research city, $\mathrm{m}$ is the number of secondary regions, is the number of administrative regions in the secondary region, and is the first-level The variance of residential prices in the administrative area is the 
variance of residential prices in the sub-regions. The value range of $q_{D, U}$ is $[0,1]$. The larger the value, the stronger the explanatory power of factor $\mathrm{D}$ on the spatial differentiation of residential prices.

\section{The Differentiation Pattern of Housing Prices in Chengdu-Chongqing Urban Agglomeration}

\subsection{Evolution of Housing Prices in Chengdu-Chongqing Urban Agglomeration}

The collected housing price data of each city in the Chengdu-Chongqing urban agglomeration from 2017 to 2019 is clustered and analyzed. According to the results, it can be seen that the housing prices of each city in the Chengdu-Chongqing urban agglomeration are mainly concentrated in $2000-8000 \mathrm{yu}-$ $\mathrm{an} / \mathrm{m}^{2}$. The county housing prices are divided into six sections from low to high, 5001 - 8000, 8001 - 10,000, 10,001 - 15,000, 15,001 - 20,000, and 20,000 - 25,000, each of which is represented by a different color, with the help of Spatial of ArcGIS software The tool draws a map of the geographic spatial distribution of housing prices in the Chengdu-Chongqing urban agglomeration in 2017, 2018, and 2019, and a map of housing price changes from 2017 to 2019 (Figure 1).

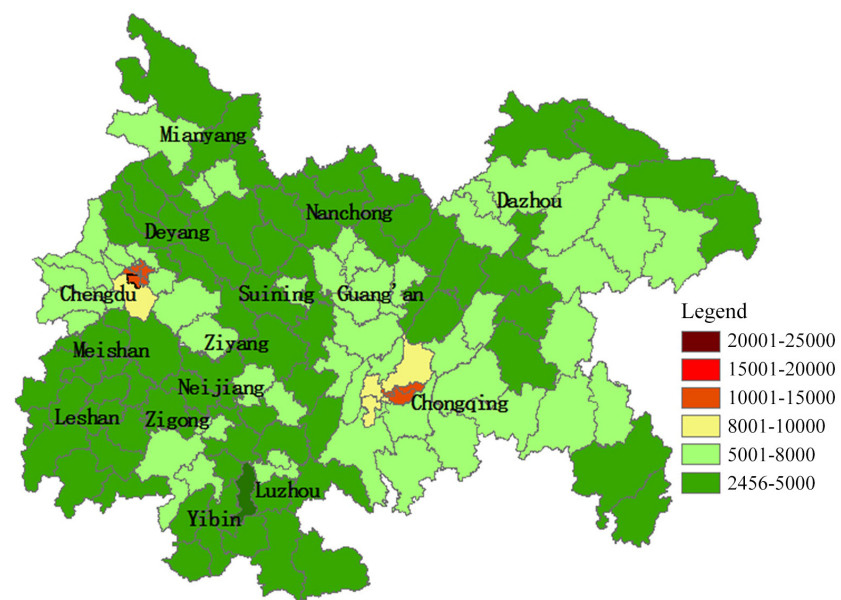

(a)

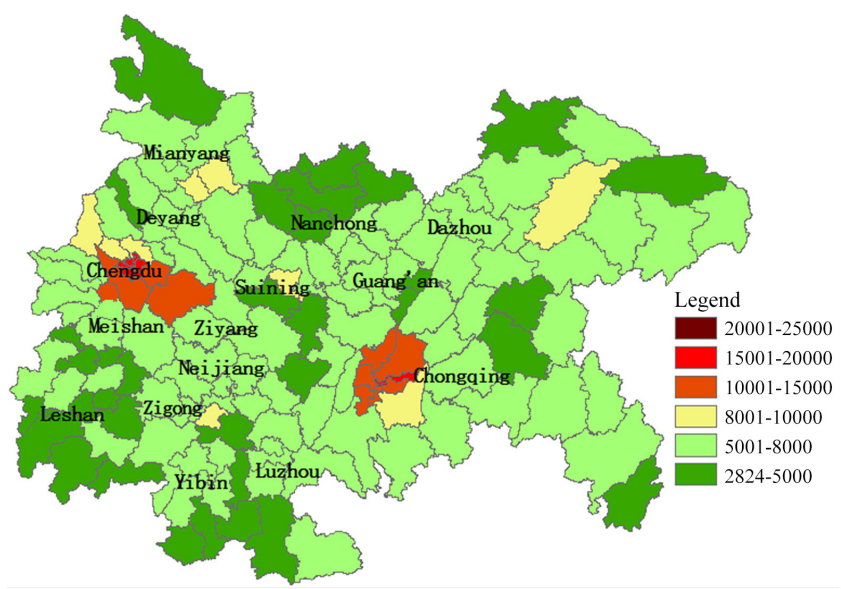

(b) 


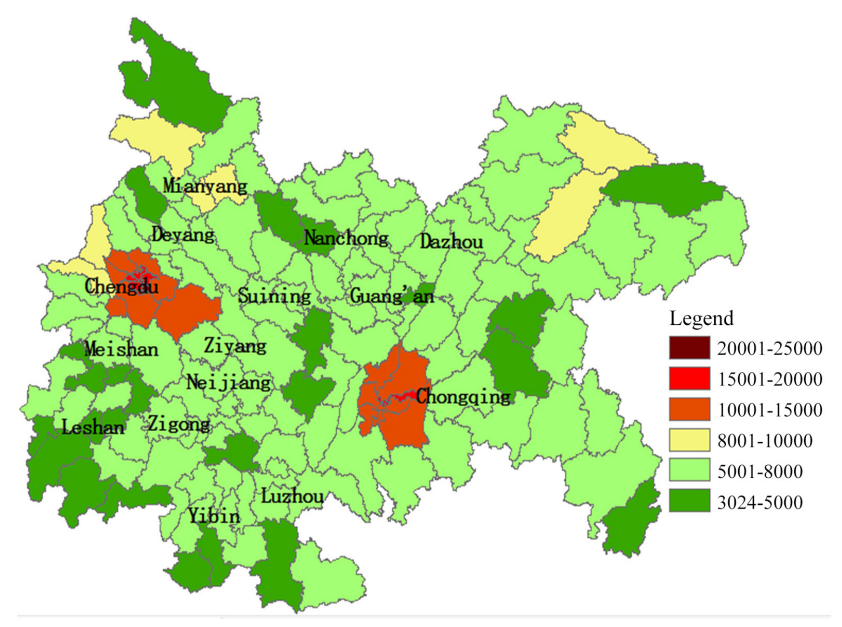

(c)

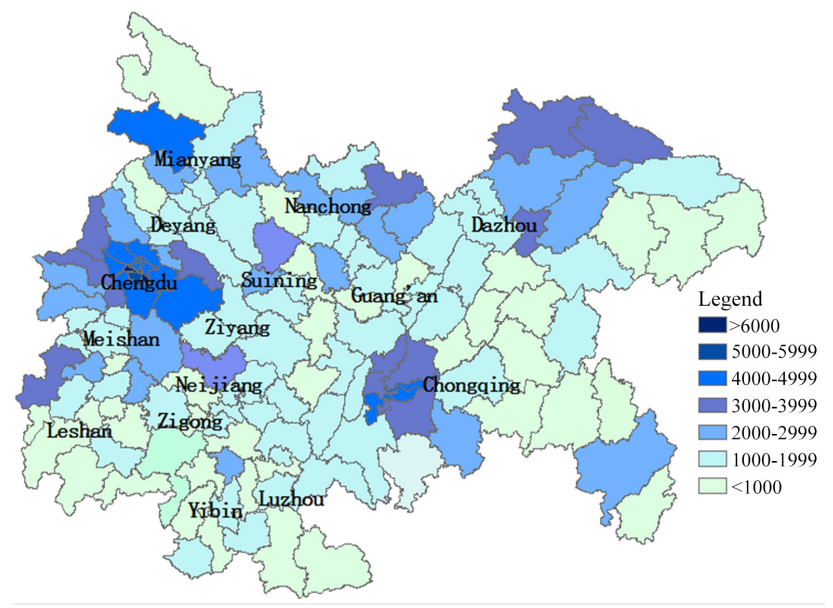

(d)

Figure 1. The geographic spatial differentiation pattern of housing prices in Chengyu urban agglomeration (2017-2019). (a) Distribution map of housing prices in Chengdu-Chongqing urban agglomeration in 2017; (b) Distribution map of housing prices in Chengdu-Chongqing urban agglomeration in 2018; (c) Distribution map of housing prices in Chengdu-Chongqing urban agglomeration in 2019; (d) Changes in housing prices in Chengdu-Chongqing urban agglomeration from 2017 to 2019.

From the 2017-2019 housing price distribution map of the Chengdu-Chongqing urban agglomeration (Figure 1), it can be seen that the housing prices of the Chengdu-Chongqing urban agglomeration are dominated by the dual cores of Chengdu and Chongqing, and the central cities have collapsed. The peripheral secondary cities are underdeveloped. Geographical space presents a severely uneven distribution. The Chengdu-Chongqing urban agglomerations in 2017, 2018, and 2019 are positively correlated with the city size and economic development of Chengdu-Chongqing area. As the cities with the highest level of development in the western region, Chengdu and Chongqing have formed Chengdu-Chongqing cities. The first tier of housing prices in the group, Mianyang, Yibin, Nanchong, and Luzhou are the second tier cities in the Chengyu 
area. The housing prices of the four cities are in the upper-middle level in the Chengdu-Chongqing area, while the housing prices of the other cities are relatively low.

Based on the housing price data of the Chengdu-Chongqing urban agglomeration in 2017 and 2019, the housing price increase from 2017 to 2019 can be calculated, and then the housing price change map can be drawn in ArcGIS (Figure 1(d)). As can be seen from the figure, the housing prices in the Chengdu-Chongqing urban agglomeration generally show an upward trend. The average housing price increase in the entire region is $33 \%$. The city with the fastest increase is still Chengdu, whose housing prices have increased from 2017 to 2019. Up to $56 \%$, the high housing prices are also located in the central urban areas of Chengdu and Chongqing. The highest average residential price in Jinjiang District of Chengdu in 2019 is as high as 20,488 yuan/ $\mathrm{m}^{2}$. The housing prices of the Chengdu-Chongqing urban agglomeration show both spatial agglomeration and administrative hierarchy. The high housing prices are mainly concentrated in the main urban areas of Chengdu and Chongqing. These areas have developed economies, complete infrastructures and mature industrial systems, which are very important to the population. In terms of administrative levels, high-priced areas are mainly distributed in provincial capital cities (municipalities and sub-provincial cities), which are discretely distributed, with medium and low housing prices concentrated in the peripheral cities of Chengdu and Chongqing. They are concentrated in prefecture-level cities, while low-price areas are distributed in blocks and counties in remote areas.

\subsection{House Price Spatial Differentiation Index Measurement}

According to formula (1), the geographical spatial differentiation index of housing prices between and within the 15 cities in the Chengdu-Chongqing urban agglomeration is calculated, and then the inter-regional and intra-regional differentiation indexes are summed to calculate the overall differentiation coefficient and inter-regional differentiation index. And the ratio of the regional differentiation index to the overall differentiation index, that is, the contribution rate of the inter-city and intra-city differentiation index to the overall differentiation degree of the Chengdu-Chongqing urban agglomeration, the calculation results are shown in Table 1 . It can be seen from the data in the table that 1) the overall housing price differentiation index of Chengyu urban agglomeration is greater than 0.3 , with a small increase and a large base, indicating that the housing price of Chengyu urban agglomeration is geographically distributed and uneven, and the spatial differentiation of housing prices still remains at At a stable and high level, the integrated and coordinated development effect of the Chengdu-Chongqing urban agglomeration is not obvious; 2) The price differentiation index among the cities in the Chengdu-Chongqing urban agglomeration is much larger than the price differentiation index within the city, indicating that the price differentiation between different cities The degree of housing price is high, and there is a large gradient of housing prices between cities; and within 15 
Table 1. Differential index and contribution rate of house price among different regions and within the Chengdu-Chongqing urban agglomeration.

\begin{tabular}{cccccc}
\hline & $\begin{array}{c}\text { Between } \\
\text { regions }\end{array}$ & $\begin{array}{c}\text { Regional } \\
\text { contribution rate }\end{array}$ & $\begin{array}{c}\text { within } \\
\text { the area }\end{array}$ & $\begin{array}{c}\text { Contribution rate } \\
\text { within the region }\end{array}$ & $\begin{array}{c}\text { Global } \\
\text { differentiation index }\end{array}$ \\
\hline $\begin{array}{c}\text { Differentiation } \\
\text { index in 2017 }\end{array}$ & 0.224 & 0.678 & 0.106 & 0.321 & 0.330 \\
$\begin{array}{c}\text { Differentiation } \\
\text { index in 2018 }\end{array}$ & 0.251 & 0.733 & 0.096 & 0.276 & 0.347 \\
$\begin{array}{c}\text { Differentiation } \\
\text { index in 2019 }\end{array}$ & 0.256 & 0.741 & 0.089 & 0.258 & 0.345 \\
\hline
\end{tabular}

cities, the housing price differentiation index is less than or equal to 0.1 , and the degree of housing price differentiation within cities is small. 3) From the point of view of contribution rate, the contribution rate of inter-regional factors to house prices from 2017 to 2019 is above 0.67 , and it is increasing year by year, indicating that inter-regional differences are still the dominant factor leading to the overall difference in housing prices in urban agglomerations and this inter-regional impact While the contribution rate in the region is below 0.32 and the contribution rate is decreasing year by year, the difference in house prices within each city has a small impact on the overall difference. The factors affecting house prices in a certain city are not necessarily the dominant factors in other cities.

\subsection{The Effect of Various Influencing Factors on the Housing Prices of Chengdu-Chongqing Urban Agglomeration Price Differences}

As an important industry in my country's rapid economic development, real estate has an interactive relationship with urban economic development. The development of urban social economy can affect urban housing prices through a variety of factors. Housing prices are subject to the compound effect of many factors, but the overall effect can be attributed to the influence of population size, land supply, national macroeconomic policies, location and other factors. This paper mainly studies the influencing factors and the mechanism of the differentiation of housing prices in Chengdu-Chongqing urban agglomerations, combined with the characteristics of the economic and social development of the Chengdu-Chongqing urban agglomeration, and selects 8 main influencing factors (economic strength, population size, real estate investment scale, higher education resources, Housing supply, housing policy, land cost, industrial structure) analyze the impact of different factors on housing prices in different cities. According to the characteristics of temporal and spatial evolution of housing prices and economic development in the Chengdu-Chongqing urban agglomeration, the Chengdu-Chongqing urban agglomeration is divided into housing price hot spots and housing price cold spots. The hot spots include Chengdu, Chongqing, Mianyang, Yibin, Deyang, and Nanchong., Luzhou, Dazhou; cold spots include Leshan City, Neijiang City, Zigong City, Suining City, 
Meishan City, Guang'an City, and Ziyang City; the index values of the 8 housing price influencing factors are divided into 5 categories from high to low: $90 \%$ 100\% (high), 70\% - 90\% (medium-high), 30\% - 70\% (medium), 10\% - 30\% (medium-low), $0 \%-10 \%$ (low), according to the proportion to determine each impact The index corresponding to the category, the discretized data of the influencing factor index value is imported into the geographic detector, and the difference in the intensity of the impact of each influencing factor on the housing price of the Chengdu-Chongqing urban agglomeration is further analyzed (Table 2). The results show that at the level of the entire Chengdu-Chongqing urban agglomeration, the value of each detection factor is quite different, which once again shows that different influencing factors have different strengths in different cities. Among them, land cost has the most significant impact, economic strength, population size, and real estate. The impact of investment scale is significant. Among all influencing factors, land cost and population size have the greatest influence on housing prices, with explanatory powers of 0.50418 and 0.34067 , respectively, indicating that at the overall level of the Chengdu-Chongqing urban agglomeration, land cost and population size play a key role in the formation of housing prices. The industrial structure and higher education resources have less power to explain the overall housing prices of the Chengdu-Chongqing urban agglomeration, which is also the shortcoming of the development of the Chengdu-Chongqing urban agglomeration.

The detection power of each influencing factor in the sub-regions divided by cold and hot spots is larger and much larger than the overall level, indicating that the selected eight influencing indicators are more explanatory for the housing prices of specific cities, and it also shows that the Chengdu-Chongqing urban agglomeration is different The development of cities has its own characteristics. In hotspots, economic strength, higher education resources, land costs, and housing purchase policies have the strongest explanatory power for housing prices, and these factors are more advantageous in the distribution of hotspots. The explanatory power of population size, housing supply, real estate investment scale, and industrial structure on housing prices is second. Among them, population size and housing supply have the least explanatory power for housing prices. This reflects that in hot cities like Chengdu and Chongqing, the real estate market towards diversification. In addition, the detection power of various

Table 2. Geographical detection results of influencing factors of residential prices in Chengdu-Chongqing city.

\begin{tabular}{ccccccccc}
\hline & $\begin{array}{c}\text { Economic } \\
\text { strength }\end{array}$ & $\begin{array}{c}\text { Population } \\
\text { size }\end{array}$ & $\begin{array}{c}\text { Real estate } \\
\text { investment } \\
\text { scale }\end{array}$ & $\begin{array}{c}\text { Higher } \\
\text { Education } \\
\text { Resources }\end{array}$ & $\begin{array}{c}\text { Housing } \\
\text { supply }\end{array}$ & $\begin{array}{c}\text { House } \\
\text { purchase } \\
\text { policy }\end{array}$ & $\begin{array}{c}\text { Land } \\
\text { cost }\end{array}$ & $\begin{array}{c}\text { Industrial } \\
\text { structure }\end{array}$ \\
\hline overall & 0.120 & 0.340 & 0.116 & 0.012 & 0.061 & 0.126 & 0.504 & 0.030 \\
Hot spot & 0.945 & 0.655 & 0.864 & 0.948 & 0.666 & 0.963 & 0.922 & 0.869 \\
Cold & & & & & & & & \\
spot & 0.543 & 0.506 & 0.161 & 0.105 & 0.188 & 0.002 & 0.487 & 0.162 \\
\hline
\end{tabular}


influencing factors in cold-spot areas is far less than that in hot-spot areas. The ability to acquire and control various resources required for economic development in cold-spot areas is much lower than in hot-spot areas. The real estate market in cold-spot areas is even more constrained by the economy, development factors and demographic factors.

\section{Conclusions and Outlook}

1) The geographical and spatial distribution of housing prices in the Chengdu-Chongqing urban agglomeration is basically consistent with the city scale and economic development level of each city. The housing prices of the Chengdu-Chongqing urban agglomeration show the characteristics of dual-core dominance and collapse in the middle. The secondary cities outside the dual-core cities are underdeveloped and there is a huge gradient in housing prices. Judging from the calculation results of the Theil index, the disparity in housing prices in the Chengdu-Chongqing urban agglomeration is increasing, which is consistent with the increasing disparity in the economic development of the Chengdu-Chongqing urban agglomeration. The differentiation of house prices among cities is the leading factor leading to the overall split of house prices in Chengdu-Chongqing urban agglomeration.

2) The leading factors for housing prices in the hot spots of the Chengdu-Chongqing urban agglomeration are economic strength, higher education resources, and housing purchase policies; while the leading factors for housing prices in cold spots are economic strength, population size, and land costs. The formation of housing prices in different regions and cities Changes are affected by basic regional characteristics such as high-quality resource allocation, urban development level, topography and climate conditions. Focusing on regional development factors and adapting measures to local conditions are important measures to further enhance the high-quality and coordinated development of the Chengdu-Chongqing urban agglomeration.

\section{Conflicts of Interest}

The authors declare no conflicts of interest regarding the publication of this paper.

\section{References}

[1] Zhang, J.H. (2019) Research on the Effect of Real Estate Economy on the Growth of China's National Economy. Fortune Today (China Intellectual Property), No. 12, 21-22.

[2] Basu, S. and Thibodeau, T.G. (1998) Analysis of Spatial Autocorrelation in House Prices. Journal of Real Estate Finance \& Economics, 17, 61-85. https://doi.org/10.1023/A:1007703229507

[3] Hanink, D.M., Cromley, R.G. and Ebenstein, A. (2012) Spatial Variation in the Determinants of House Prices and Apartment Rents in China. Journal of Real Estate Finance and Economics, 45, 347-363. https://doi.org/10.1007/s11146-010-9262-3 
[4] Holmes, M.J., Otero, J. and Panagiotidis, T. (2019) Property Heterogeneity and Convergence Club Formation among Local House Prices. Journal of Housing Economics, 43, 1-13. https://doi.org/10.1016/j.jhe.2018.09.002

[5] Cui, N.N., Gu, H.Y. and Shen, T.Y. (2019) Spatial Differentiation and Correlation of Housing Prices and Rents in Beijing. Geographical Research, 38, 1420-1434.

[6] Shi, Y.S. and Li, M.X. (2006) Analysis of the Housing Price Gradient and Its Influencing Factors in Shanghai. Acta Geographica Sinica, 61, 604-612.

[7] Hu, X.T., Pu, L.J. and Pan, J. (2006) Study on the Spatiotemporal Impact of Urban Land Market on Housing Prices: Based on the Comparative Analysis of Nanjing and Guangzhou. Journal of Jiangxi Agricultural University, 28, 793-796.

[8] Song, W.X. and Liu, C.H. (2018) Research on the Price Differentiation Mechanism of Urban Commercial Housing in the Integrated Area of the Yangtze River Delta. Geographical Research, 37, 92-102.

[9] Wang, Y., Wang, D.L. and Wang, S.J. (2013) The Spatial Differentiation Pattern and Influencing Factors of Urban Housing Prices in China. Geographical Sciences, No. 10, 3-11.

[10] Wang, S.J., Wang, Y., Lin, X.Q., et al. (2016) The Characteristics and Influencing Mechanism of Spatial Differences in Housing Prices in Counties in China. Acta Geographica Sinica, No. 8, 1329-1342.

[11] Han, Y.H., Yin, S.G. and Li, Z.J. (2018) Analysis of the Spatial Differentiation Pattern of Housing Prices in Counties in the Yangtze River Delta and Its Influencing Factors. Human Geography, 33, 93-101.

[12] Mao, Z.G. and Wu, Y.M. (2020) Analysis of the Spatial Structure Evolution of the Consumption Level of Urban Agglomerations in Western China: Taking Chengyu Urban Agglomeration as an Example. Journal of Xiangtan University, 2, 50-55.

[13] Wang, J.F., Li, X.H., Christakos, G., et al. (2010) Geographical Detectors-Based Health Risk Assessment and Its Application in the Neural Tube Defects Study of the Heshun Region, China. International Journal of Geographical Information Science, 24, 107-127. https://doi.org/10.1080/13658810802443457 